\title{
Gauss Legendre-Gauss Jacobi quadrature rules over a tetrahedral region
}

\author{
H.T. Rathod ${ }^{\text {a }}$, B. Venkatesudu ${ }^{\text {b,* }}$, K.V. Nagaraja ${ }^{\text {b }}$, Md. Shafiqul Islam ${ }^{\text {c }}$ \\ a Department of Mathematics, Central College Campus, Bangalore University, Bangalore 560001 , India \\ b Department of Mathematics, Amrita School of Engineering, Bangalore 560 035, India \\ c Department of Mathematics, University of Dhaka, Dhaka 1000, Bangladesh
}

\begin{abstract}
This paper presents a Gaussian Quadrature method for the evaluation of the triple integral $\iint_{T} \int f(x, y, z) \mathrm{d} x \mathrm{~d} y \mathrm{~d} z$, where $f(x, y, z)$ is an analytic function in $x, y, z$ and $T$ refers to the standard tetrahedral region: $\{(x, y, z) \mid 0 \leqslant x, y, z \leqslant$ $1, x+y+z \leqslant 1\}$. in three space $(x, y, z)$. Mathematical transformation from $(x, y, z)$ space to $(U, V, W)$ space map the standard tetrahedron $T$ in $(x, y, z)$ space to a standard 1-cube: $\{(U, V, W) / 0 \leqslant U, V, W \leqslant 1\}$ in $(U, V, W)$ space. Then we use the product of Gauss Legendre and Gauss Jacobi weight coefficients and abscissas to arrive at an efficient quadrature rule over the standard tetrahedral region $T$. We have then demonstrated the application of the derived quadrature rules by considering the evaluation of some typical triple integrals over the region $T$.
\end{abstract}

(c) 2007 Elsevier Inc. All rights reserved.

Keywords: Finite element method; Gauss Legendre-Gauss Jacobi quadrature rules; Tetrahedral regions; Standard 1-cube; Orthogonal polynomials

\section{Introduction}

In recent years, we have been witnessing finite element method (FEM) gaining importance due to the most obvious reason that it can provide solutions to many complicated problems that would be intractable by other numerical techniques [1,2]. In FEM it may be possible to perform some of the integration analytically, particularly if constant or linear elements are used to discretise the surface or boundary curve of the given region. However, with higher order elements or for more complex distorted elements the integrals become too complicated for analytical integration and the numerical integration is essential, among various integration schemes, Gauss Legendre quadrature which can evaluate exactly the $(2 n-1)$ th order polynomial with $n$-Gaussian points is most commonly used in view of the accuracy and efficiency of calculations [3]. The triangular and tetrahedral elements are very widely used in finite element analysis. The versatility of these

\footnotetext{
* Corresponding author.

E-mail address: venkateshoxford1234@yahoo.com (B. Venkatesudu).
} 
elements can be further enhanced by improved numerical integration schemes. Mathematically, the problem can be defined as the evaluation of the following integrals

$$
\mathrm{II}=\int_{0}^{1} \int_{0}^{1-L_{1}} F\left(L_{1}, L_{2}, L_{3}\right) \mathrm{d} L_{2} \mathrm{~d} L_{1}
$$

where $L_{1}, L_{2}, L_{3}$ are the well known area co-ordinates and

$$
\mathrm{III}=\int_{0}^{1} \int_{0}^{1-L_{1}} \int_{0}^{1-L_{1}-L_{2}} G\left(L_{1}, L_{2}, L_{3}, L_{4}\right) \mathrm{d} L_{3} \mathrm{~d} L_{2} \mathrm{~d} L_{1},
$$

where $L_{1}, L_{2}, L_{3}, L_{4}$ are the well known volume co-ordinates.

The basic problem of integrating an arbitrary function of two variables over the surface of the triangle were first given by Hammer et al. [4] and Hammer and Stroud [5,6]. Cowper [7] provided a table of Gaussian quadrature formulae with symmetrically placed integration points. Lyness and Jespersen [8] made an elaborate study of symmetric quadrature rules by formulating the problem in polar co-ordinates. Lannoy [9] discussed the symmetric 4-point integration formula, which is presented in [7]. Laurie [10] derived a 7-point integration rule and discussed the numerical error in integrating some functions. Laursen and Gellert [11] gave a table of symmetric integration formulae up to a precision of degree ten. Dunavant [12] presented some extensions to the integration formulae given by Lyness and Jespersen [8] and also gave tables of integration formulae with precisions of degree from 11 to 20. Sylvester [13] derived some numerical integration formulae for triangles as product of one-dimensional Newton Cotes rules of closed type as well as open type. The precision of these integration formulae is limited to a degree ten atmost for various reasons. Lether [14] and Hillion [15] derived the formulae for triangles as product of one-dimensional Gauss Legendre and Gauss Jacobi quadrature rules. The precision of these formulae is again up to degree seven. This is because the zeros and weight coefficients of Gauss Jacobi orthogonal polynomials with weight functions $x, x^{2}, x^{3}$ were available for polynomials of degree up to six only. Even today the zeros and weights for the integral $\int_{0}^{1} x^{r} f(x) \mathrm{d} x, r=0,1,2$ are not available beyond a formula of order-eight as documented in Abramowicz and Stegun [16]. Reddy [17] and Reddy and Shippy [18] derived the 3-point, 4-point, 6-point and 7-point rules of precision 3, 4, 6 and 7, respectively which gave improved accuracy. Since the precision of all the formulae derived by the authors [4-18] is limited to a precision of degree ten and it is not likely that the techniques can be extended much further to give a greater accuracy which may be demanded in future, Lague and Baldur [19] proposed the product formulae based only on the sampling points and weight coefficients of Gauss Legendre quadrature rules. By the proposed method of [19] this restriction is removed and one can now obtain numerical integration rules of very high degree of precision as the derivation now rely on standard Gauss Legendre Quadarature rules. However, the Lague and Baldur [19] have not worked out explicit weight coefficients and sampling points for application to integrals over a triangular surface. Rathod et al. [20-22] provided this information in a systematic manner in their recent work. For tetrahedral regions, four volume coordinates $L_{1}, L_{2}, L_{3}, L_{4}$ are involved and we have to compute numerically the integral III stated in Eq. (2). Numerical integration formulae for III with a degree of precision $d=1,2,3$ are listed in Zienkiewicz [1] and these are based on reference [4]. Rathod et al. [23] proposed product formulae based on Gauss Legendre quadrature Rule for the numerical integration of an arbitrary function over the standard tetrahedron. In this paper, we propose the product rule based on Gauss Legendre-Gauss Jacobi quadrature rules which has a higher precision than our earlier work [23], and are based on zeros and weight coefficients of the Gauss Legendre-Gauss Jacobi quadrature rules $\int_{0}^{1} x^{r} \Phi(x) \mathrm{d} x$, $r=0,1,2$.

\section{Gauss quadrature formulas}

Given any $n$ distinct points

$$
-1 \leqslant x_{1}<x_{2}<\cdots<x_{n-1}<x_{n} \leqslant 1
$$

and weight function $w(x)$ which is positive and integrable on the interval [-1,1], we can find constants $A_{1}, A_{2}, \ldots, A_{n}$ so that the formula 


$$
\int_{-1}^{1} f(x) w(x) \mathrm{d} x=\sum_{k=1}^{n} A_{K} f\left(x_{k}\right)+E(f)
$$

is exact (that is $E(f))=0$ ) whenever $f(x)$ is a polynomial of degree $\leqslant(n-1)$. But it was Gauss [24] who proved that for a certain special choice of $x_{1}, x_{2}, \ldots, x_{n}$, we can find $A_{1}, A_{2}, \ldots, A_{n}$ so that the formula (4) has degree of exactness equal to $(2 n-1)$, (i.e., $E(f))=0$ if $\mathrm{f}$ is a polynomial of degree $\leqslant(2 n-1)$ ). Let $w(x)=(1-x)^{\alpha}(1+x)^{\beta},(\alpha, \beta>-1)$ be the weight functions. These weight functions are known as Jacobi weights and the corresponding orthogonal polynomials are known as Jacobi polynomials $P_{n}^{(\alpha, \beta)}(x)$. Let $x_{1}, x_{2}, \ldots, x_{n}$ be the zeros of the $n$th degree Jacobi Polynomials $P_{n}^{(\alpha, \beta)}(x)$. It is well known (see [25, p. 349]) that the corresponding Gaussian Quadrature formula is given by

$$
\begin{aligned}
& \left(f \in \pi_{2 n-1}\right)=\int_{-1}^{1} w(x) f(x) \mathrm{d} x=\sum_{k=1}^{n} f\left(x_{k}\right) A_{K}^{(\alpha, \beta)}+E_{2 n-1}(f), \\
& \text { where } A_{k}^{(\alpha, \beta)}=\frac{2^{\alpha+\beta+1} \Gamma(\alpha+n+1) \Gamma(\beta+n+1)}{\Gamma(n+1) \Gamma(\alpha+\beta+n+1)\left(1-x_{k}^{2}\right)\left[P_{n}^{(\alpha, \beta) \prime}(x)\right]^{2}}, \\
& (1-x)^{\alpha}(1+x)^{\beta} P_{n}^{(\alpha, \beta)}(x)=\frac{(-1)^{n}}{2^{n} n !} \frac{\mathrm{d}^{n}}{\mathrm{~d} x^{n}}\left[(1-x)^{n+\alpha}(1+x)^{n+\beta}\right] .
\end{aligned}
$$

In this paper, we need integrals over $(0,1)$, and hence we shall refer to the Gauss Jacobi rules derived in Villadsen and Michelson [26], though it is possible for us to derive these from the formulas quoted in Eqs. (5)-(7). The formulas can be very systematically generated from [26] as given in the following:

$$
\int_{0}^{1}(1-x)^{\alpha} x^{\beta} f(x) d x=\sum_{i=1}^{n} w_{i}^{(\alpha, \beta)} f\left(x_{i}\right)+E_{2 n-1}(f),
$$

where $x_{i}^{\prime} s$ are the zeros of $P_{N}^{(\alpha, \beta)}(x)$ over $(0,1)$ and

$$
\begin{aligned}
P_{N}^{(\alpha, \beta)}(x) & =\frac{(-1)^{N} \Gamma(\beta+1)}{\Gamma(N+\beta+1)}(1-x)^{-\alpha} x^{-\beta} \frac{d^{N}}{d x^{N}}\left[(1-x)^{N+\alpha} x^{N+\beta}\right] \\
& =\sum_{i=0}^{N}(-1)^{N-i} \gamma_{N, i}^{(\alpha, \beta)} x^{i}, \\
\gamma_{N, i}^{(\alpha, \beta)}= & \frac{(N-i+1)}{i} \frac{(N+\alpha+\beta+i)}{(\beta+i)} \gamma_{N, i-1}^{(\alpha, \beta)}, \quad i=1,2, \ldots, N, \\
\gamma_{N, 0}^{(\alpha, \beta)}=1 &
\end{aligned}
$$

and the weight coefficients $w_{i}^{(\alpha, \beta)}$ are given by:

$$
\begin{aligned}
& w_{i}^{(\alpha, \beta)}=\frac{(2 N+\alpha+\beta+1)}{x_{i}\left(1-x_{i}\right)} \frac{C_{N}^{(\alpha, \beta)}}{\left[P_{N}^{(1)(\alpha, \beta)}\left(x_{i}\right)\right]^{2}}, \\
& P_{N}^{(\alpha, \beta)}(x)=\frac{P_{N}^{(\alpha, \beta)}(x)}{\gamma_{N, N}^{(\alpha, \beta)}}, \\
& \gamma_{N, N}^{(\alpha, \beta)}=\frac{\Gamma(2 N+\alpha+\beta+1)}{\Gamma(N+\alpha+\beta+1) \Gamma(N+\beta+1)}, \\
& C_{N}^{(\alpha, \beta)}=\frac{\Gamma(N+\alpha+\beta+1) \Gamma(N+\beta+1) \Gamma(N+1) \Gamma(N+\alpha+1)}{\{\Gamma(2 N+\alpha+\beta+1)\}^{2}(2 N+\alpha+\beta+1) .}
\end{aligned}
$$

We shall now present the special case of formulas of Eq. (8) for $\alpha=0$. This gives us the following Gauss Jacobi quadrature rules $(\alpha=0, \beta=0$, refers to the Gauss Legendre quadrature rule over $(0,1))$ :

$$
\int_{0}^{1} x^{\beta} f(x) \mathrm{d} x=\sum_{i=1}^{n} w_{i}^{(0, \beta)} f\left(x_{i}\right)+E_{2 n-1}(f),
$$


where $x_{i}^{\prime} s$ are the zeros of $P_{N}^{(0, \beta)}(x)$ over $(0,1)$ and

$$
\begin{aligned}
& P_{N}^{(0, \beta)}(x)=\frac{(-1)^{N} \beta !}{(N+\beta) !} \sum_{k=0}^{N}(-1)^{k} C_{k}^{N} \frac{(N+\beta+k) !}{(\beta+k) !} x^{k} \\
& =\sum_{k=0}^{N}(-1)^{N-k} \gamma_{k}^{N} x^{k}, \\
& \gamma_{k}^{N}=\frac{(N-k+1)}{k} \frac{(N+\beta+k)}{(\beta+k)} \gamma_{k-1}^{N}, \quad k=1,2, \ldots, N, \\
& \gamma_{0}^{N}=1
\end{aligned}
$$

and the weight coefficients $w_{i}^{(0, \beta)}$ are given by

$$
w_{i}^{(0, \beta)}=\frac{(2 N+\beta+1)}{x_{i}\left(1-x_{i}\right)} \frac{C_{N}^{(0, \beta)}}{\left[P_{N}^{(1)(0, \beta)}\left(x_{i}\right)\right]^{2}},
$$

where

$$
C_{N}^{(0, \beta)}=\frac{\left[\frac{N !(N+\beta) !}{(2 N+\beta) !}\right]^{2}}{(2 N+\beta+1)} .
$$

If necessary, one can find the abscissas and weight coefficients for higher order rules by the procedure outlined in Eqs. (12)-(16) and computer programs already available in [26].

\section{Formulation of integrals over a tetrahedron}

The finite element method for three-dimensional problems with tetrahedron element requires the numerical integration of expressions containing product of shape functions and their global derivatives over a standard tetrahedron $T$ with coordinates $(0,0,0),(1,0,0),(0,1,0)$ and $(0,0,1)$ in the natural coordinate space $(x, y, z)$ say. The numerical integration of an arbitrary function $f$, over the tetrahedron $T$ is given by

$$
\begin{aligned}
I & =\iiint_{T} f(x, y, z) \mathrm{d} x \mathrm{~d} y \mathrm{~d} z=\int_{0}^{1} \mathrm{~d} x \int_{0}^{1-x} \mathrm{~d} y \int_{0}^{1-x-y} f(x, y, z) \mathrm{d} z \\
& =\int_{0}^{1} \mathrm{~d} y \int_{0}^{1-y} \mathrm{~d} x \int_{0}^{1-x-y} f(x, y, z) \mathrm{d} z .
\end{aligned}
$$

It is now required to find the value of the integral by a quadrature formula:

$$
I=\sum_{m=1}^{N} c_{m} f\left(x_{m}, y_{m}, z_{m}\right),
$$

where $c_{m}$ are the weights associated with the sampling points $\left(x_{m}, y_{m}, z_{m}\right)$ and $N$ is the number of pivotal points related to the required precision.

The integral $I$ of Eq. (17) can be transformed into an integral over the cube: $\{(u, v, w) \mid 0 \leqslant u, v, w \leqslant 1\}$ by the substitution

$$
x=u, y=(1-u) v, z=(1-u)(1-v) w .
$$

Then the determinant of the Jacobian and the differential volume are:

$$
\begin{aligned}
& \frac{\partial(x, y, z)}{\partial(u, v, w)}=\left|\begin{array}{lll}
\frac{\partial x}{\partial u} & \frac{\partial x}{\partial v} & \frac{\partial x}{\partial w} \\
\frac{\partial y}{\partial u} & \frac{\partial y}{\partial v} & \frac{\partial y}{\partial w} \\
\frac{\partial z}{\partial u} & \frac{\partial z}{\partial v} & \frac{\partial z}{\partial w}
\end{array}\right|=(1-u)^{2}(1-v) \text { and } \\
& \mathrm{d} x \mathrm{~d} y \mathrm{~d} z=\frac{\partial(x, y, z)}{\partial(u, v, w)} \mathrm{d} u \mathrm{~d} v \mathrm{~d} w=(1-u)^{2}(1-v) \mathrm{d} u \mathrm{~d} v \mathrm{~d} w
\end{aligned}
$$


Then on using Eqs. (19) and (20) in Eq. (17), we have

$$
\begin{aligned}
I & =\int_{0}^{1}\left(\int_{0}^{1-x}\left(\int_{0}^{1-x-y} f(x, y, z) \mathrm{d} z\right) \mathrm{d} y\right) \mathrm{d} x \\
& =\int_{0}^{1} \int_{0}^{1} \int_{0}^{1} f(u,(1-u) v,(1-u)(1-v) w) \times(1-u)^{2}(1-v) \mathrm{d} u \mathrm{~d} v \mathrm{~d} w .
\end{aligned}
$$

In Eq. (21), let us substitute

$$
U=1-u, \quad V=1-v, \quad W=w
$$

so that, we then obtain:

$$
I=\int_{0}^{1} \int_{0}^{1} \int_{0}^{1} f[1-U, U(1-V), U V W] \times U^{2} V \mathrm{~d} U \mathrm{~d} V \mathrm{~d} W .
$$

Eq. (23) represents an integral over the standard 1-cube: $\{(U, V, W) \mid 0 \leqslant U, V, W \leqslant 1\}$. We shall now use the Gauss Legendre-Gauss Jacobi Quadrature rules described in the previous Section 2 and developed in Eqs. (12)-(16) to approximate the triple integral of Eq. (23) above. We thus have

$$
I \simeq \sum_{i=1}^{\alpha} \sum_{j=1}^{\beta} \sum_{k=1}^{\gamma} \lambda_{i}^{(\alpha)} \mu_{j}^{(\beta)} v_{k}^{(\gamma)} \times f\left[1-U_{i}^{(\alpha)}, U_{i}^{(\alpha)}\left(1-V_{j}^{(\beta)}\right), U_{i}^{(\alpha)} V_{j}^{(\beta)} W_{k}^{(\gamma)}\right]=\sum_{m=1}^{N=(\alpha \times \beta \times \gamma)} c_{m} f\left(x_{m}, y_{m}, z_{m}\right),
$$

where it is obvious that

$$
c_{m}=\lambda_{i}^{(\alpha)} \mu_{j}^{(\beta)} v_{k}^{(\gamma)}, \quad x_{m}=1-U_{i}^{(\alpha)}, \quad y_{m}=U_{i}^{(\alpha)}\left(1-V_{j}^{(\beta)}\right), \quad z_{m}=U_{i}^{(\alpha)} V_{j}^{(\beta)} W_{k}^{(\gamma)},
$$

in which the pairs $\left(\lambda_{i}^{(\alpha)}, U_{i}^{(\alpha)}\right),\left(\mu_{j}^{(\beta)}, V_{j}^{(\beta)}\right)$ and $\left(v_{k}^{(\gamma)}, W_{k}^{(\gamma)}\right)$ refer to the abscissas and weight coefficients of Gauss Legendre and Gauss Jacobi Quadrature rules over $(0,1)$ of order $\alpha, \beta$ and $\gamma$ respectively for the integrals $\int_{0}^{1} x^{r} f(x) \mathrm{d} x, r=0,1,2$. In the present analysis we shall assume $\alpha=\beta=\gamma$.

The weight coefficients $c_{m}$ and corresponding sampling points $\left(x_{m}, y_{m}, z_{m}\right)$ of various orders can be now easily computed by the formulas of Eq. (25) and the approximation to the integral $I$ can be then computed by Eq. (24). We have listed here $C$-Program which generates $c_{m},\left(x_{m}, y_{m}, z_{m}\right)$ and then compute the integral $I=\iint_{T} \int f(x, y, z) \mathrm{d} x \mathrm{~d} y \mathrm{~d} z$ for some sample functions $f(x, y, z)$.

3.1. C-Program for generating sampling points $\left(x_{m}, y_{m}, z_{m}\right)$, weight coefficients $\left(c_{m}\right)$ and evaluation of triple integrals of Examples 1 and 2

\#include $<$ stdio.h $>$

\#include $<$ conio.h $>$

\#include $<$ math.h $>$

main()

\{

int $\mathrm{i}, \mathrm{j}, \mathrm{k}, \mathrm{n}$;

double

$\mathrm{xm}[1000], \mathrm{ym}[1000], \mathrm{zm}[1000], \mathrm{cm}[1000], \mathrm{a}[1000], \mathrm{b}[1000], \mathrm{c}[1000], \mathrm{w} 1[1000], \mathrm{w} 2[1000], \mathrm{w} 3[1000] ;$

double $\mathrm{I} 1=0.0, \mathrm{I} 2=0.0, \mathrm{I} 3=0.0, \mathrm{I} 4=0.0, \mathrm{I} 5=0.0$;

clrscr();

printf("Enter the value of $\mathrm{n}=$ ");

scanf("\%d", \&n);

printf("Enter the values of alphas (a, b, c's)");

$/ /$ for $(\mathrm{i}=1 ; \mathrm{i}<=\mathrm{n} ; \mathrm{i}++)$

$/ /$ for $(\mathrm{j}=1 ; \mathrm{j}<=\mathrm{n} ; \mathrm{j}++)$ 


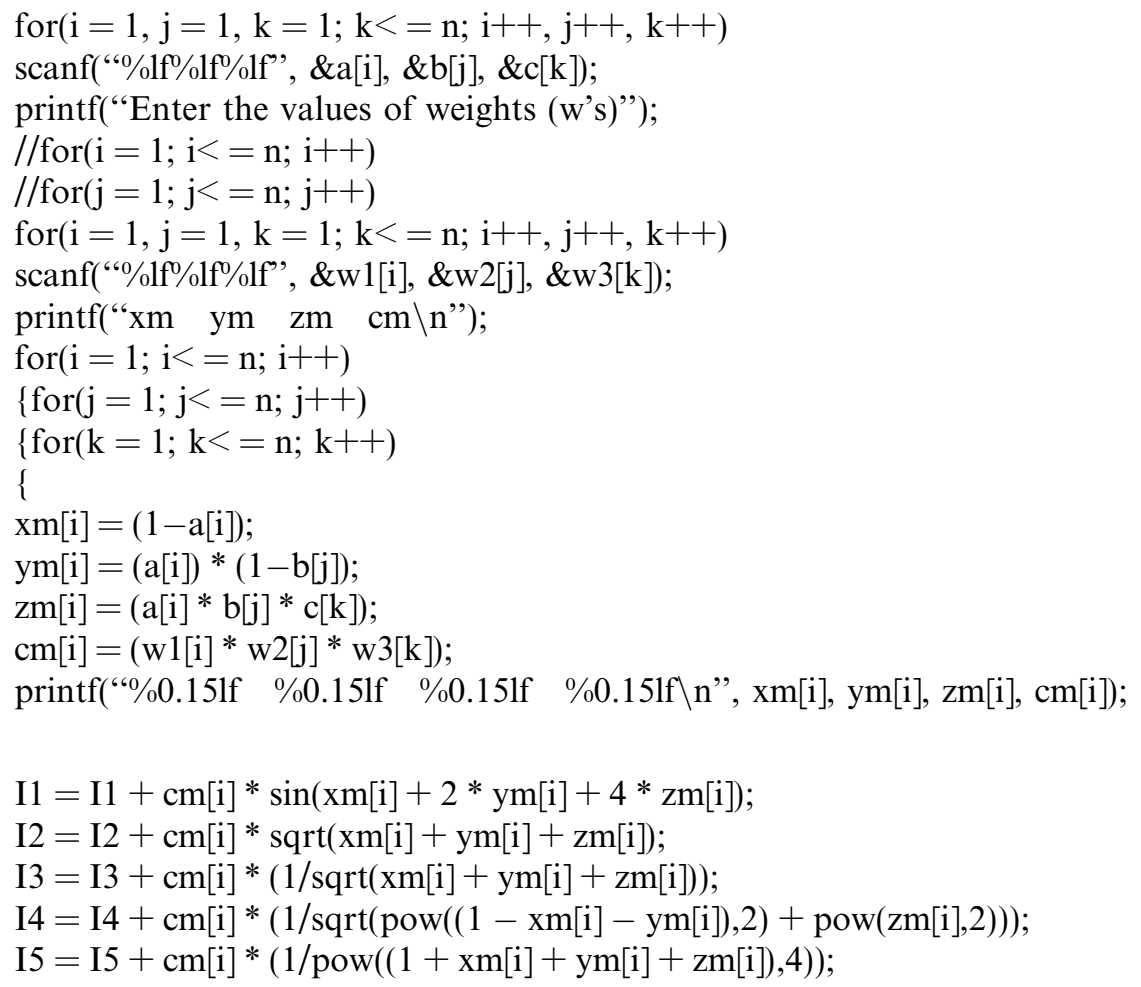

\}\}\} printf("Sum I1 $=\% 0.151 f \backslash n \quad$ Sum $\quad$ I2 $=\% 0.151 f \backslash n \quad$ Sum $\quad I 3=\% 0.151 f \backslash n \quad$ Sum $\quad$ I $4=\% 0.151 f \backslash n \quad$ Sum $\mathrm{I} 5=\% 0.15 \mathrm{lf} \backslash \mathrm{n} ”, \mathrm{I} 1, \mathrm{I} 2, \mathrm{I} 3, \mathrm{I} 4, \mathrm{I} 5)$;

$\operatorname{getch}()$;

\}

3.2. Sample output for $n=2$ and 3

\begin{tabular}{llll}
\hline$x_{m}$ & $y_{m}$ & $z_{m}$ & $c_{m}$ \\
\hline$n=2$ & & & \\
0.544151844000000 & 0.293998800648746 & 0.034202793234680 & 0.009169429925531 \\
0.544151844000000 & 0.293998800648746 & 0.127646562116573 & 0.009169429925531 \\
0.544151844000000 & 0.070679724151254 & 0.081395667016765 & 0.016027040599469 \\
0.544151844000000 & 0.070679724151254 & 0.303772764831981 & 0.016027040599469 \\
0.122514822700000 & 0.565933165063089 & 0.065838687051064 & 0.021157006463867 \\
0.122514822700000 & 0.565933165063089 & 0.245713325185847 & 0.021157006463867 \\
0.122514822700000 & 0.136054976776911 & 0.156682637329037 & 0.036979856361133 \\
0.122514822700000 & 0.136054976776911 & 0.584747563194052 & 0.036979856361133 \\
& & & \\
$n=3$ & & & \\
0.705002209900000 & 0.232357800582355 & 0.007059631139277 & 0.000580935315705 \\
0.705002209900000 & 0.232357800582355 & 0.031319994758822 & 0.000929496504961 \\
0.705002209900000 & 0.232357800582355 & 0.055580358378368 & 0.000580935315705
\end{tabular}


Table (continued)

\begin{tabular}{llll}
\hline$x_{m}$ & $y_{m}$ & $z_{m}$ & $c_{m}$ \\
\hline 0.705002209900000 & 0.120791820117176 & 0.019633302939687 & 0.001907203414924 \\
0.705002209900000 & 0.120791820117176 & 0.087102984991412 & 0.003051525463329 \\
0.705002209900000 & 0.120791820117176 & 0.154572667043137 & 0.001907203414924 \\
0.705002209900000 & 0.026133252281968 & 0.030301481179093 & 0.001671681130814 \\
0.705002209900000 & 0.026133252281968 & 0.134432268909016 & 0.002674689808822 \\
0.705002209900000 & 0.026133252281968 & 0.238563056638938 & 0.001671681130814 \\
0.347003766000000 & 0.514338662229867 & 0.015626939258814 & 0.002836648696577 \\
0.347003766000000 & 0.514338662229867 & 0.069328785885067 & 0.004538637913707 \\
0.347003766000000 & 0.514338662229867 & 0.123030632511319 & 0.002836648696577 \\
0.347003766000000 & 0.267380320400989 & 0.043459555667351 & 0.009312682384413 \\
0.347003766000000 & 0.267380320400989 & 0.192807956799506 & 0.014900291812379 \\
0.347003766000000 & 0.267380320400989 & 0.342156357931660 & 0.009312682384413 \\
0.347003766000000 & 0.057847603931245 & 0.067074241769277 & 0.008162650767857 \\
0.347003766000000 & 0.057847603931245 & 0.297574315034378 & 0.013060241226220 \\
0.347003766000000 & 0.057847603931245 & 0.528074388299478 & 0.008162650767857 \\
0.072994024100000 & 0.730165028062778 & 0.022184302640169 & 0.003047877091380 \\
0.072994024100000 & 0.730165028062778 & 0.098420473918611 & 0.004876603345330 \\
0.072994024100000 & 0.730165028062778 & 0.174656645197053 & 0.003047877091380 \\
0.072994024100000 & 0.379578230231835 & 0.061696018622970 & 0.010006142576977 \\
0.072994024100000 & 0.379578230231835 & 0.273713872834082 & 0.016009828120281 \\
0.072994024100000 & 0.379578230231835 & 0.485731727045195 & 0.010006142576977 \\
0.072994024100000 & 0.082121567849287 & 0.095219879857808 & 0.008770474930612 \\
0.072994024100000 & 0.082121567849287 & 0.422442204025356 & 0.014032759886453 \\
0.072994024100000 & 0.082121567849287 & 0.749664528192904 & 0.008770474930612 \\
\hline
\end{tabular}

\section{Some numerical results}

We consider some typical integrals with known exact values

Example 1. Let us consider the following multiple integrals which are generalised to three-dimensions from Reddy and Shippy [18].

$$
\begin{aligned}
& I_{1}=\int_{0}^{1} \int_{0}^{1-x} \int_{0}^{1-x-y} \sqrt{(x+y+z)} \mathrm{d} z \mathrm{~d} y \mathrm{~d} x=0.142857142857143 \\
& I_{2}=\int_{0}^{1} \int_{0}^{1-x} \int_{0}^{1-x-y} \frac{\mathrm{d} z \mathrm{~d} y \mathrm{~d} x}{\sqrt{(x+y+z)}}=0.200000000000000 \\
& I_{3}=\int_{0}^{1} \int_{0}^{1-x} \int_{0}^{1-x-y}\left[(1-x-y)^{2}+z^{2}\right]^{\frac{-1}{2}} \mathrm{~d} z \mathrm{~d} y \mathrm{~d} x=0.440686793509772 .
\end{aligned}
$$

Example 2. We now consider the following multiple integrals from Stroud [6].

$$
\begin{aligned}
& I_{4}=\int_{0}^{1} \int_{0}^{1-x} \int_{0}^{1-x-y} \sin (x+2 y+4 z) \mathrm{d} z \mathrm{~d} y \mathrm{~d} x=0.131902326890181
\end{aligned}
$$

We have tabulated the numerical values for $I_{1}, I_{2}$ and $I_{3}$ of Examples 1 and $I_{4}$ and $I_{5}$ of Examples 2, in Tables 1 and 2 respectively. 
Table 1

Numerical results for triple integrals of Example 1

\begin{tabular}{llll}
\hline$n^{3}$ & $I_{1}$ & $I_{2}$ & $I_{3}$ \\
\hline $2^{3}$ & 0.142922197113895 & 0.198983291341586 & 0.381020286857648 \\
$3^{3}$ & 0.142864935725559 & 0.199761492149967 & 0.408992760975680 \\
$4^{3}$ & 0.142858682579356 & 0.199922912518858 & 0.421179840342725 \\
$5^{3}$ & 0.142857556536540 & 0.199969372785542 & 0.427473953838342 \\
$6^{3}$ & 0.142857279888475 & 0.199985955383987 & 0.431142555206647 \\
$7^{3}$ & 0.142857173245610 & 0.199995955453215 & 0.434255520664723 \\
$8^{3}$ & 0.142857152996523 & 0.199996036650226 & 0.435031208274682 \\
$9^{3}$ & 0.142857152535564 & 0.199997676631442 & 0.436135693229094 \\
\hline
\end{tabular}

Table 2

Numerical results for triple integrals of Example 2

\begin{tabular}{lll}
\hline$n^{3}$ & $I_{4}$ & $I_{5}$ \\
\hline $2^{3}$ & 0.130611579439609 & 0.020645478380531 \\
$3^{3}$ & 0.131927425607066 & 0.020825042317841 \\
$4^{3}$ & 0.131902030920072 & 0.020833013963470 \\
$5^{3}$ & 0.131902328985835 & 0.020833321868462 \\
$6^{3}$ & 0.131902326836479 & 0.020833332921807 \\
$7^{3}$ & 0.131902324856213 & 0.020833332245126 \\
$8^{3}$ & 0.131902314247159 & 0.020833332255096 \\
$9^{3}$ & 0.131902325772292 & 0.020833333237911 \\
\hline
\end{tabular}

\section{Conclusions}

In this paper we have derived Gaussian Quadrature method for the evaluation of the triple integral $\iint_{T} \int f(x, y, z) \mathrm{d} x \mathrm{~d} y \mathrm{~d} z$, where $f(x, y, z)$ is an analytic function in $x, y, z$ and $T$ refers to the standard tetrahedral region: $\{(x, y, z) \mid 0 \leqslant x, y, z \leqslant 1, x+y+z \leqslant 1\}$. in three space $(x, y, z)$.

Mathematical transformation from $(x, y, z)$ space to $(U, V, W)$ space map the standard tetrahedron $T$ in $(x, y, z)$ space to a standard 1-cube: $\{(U, V, W) / 0 \leqslant U, V, W \leqslant 1\}$ in $(U, V, W)$ space. Then we use the product of Gauss Legendre and Gauss Jacobi weight coefficients and abscissas to arrive at an efficient quadrature rule over the standard tetrahedral region $T$. We have then demonstrated the application of the derived quadrature rules by considering the evaluation of some typical triple integrals over the region $T$.

\section{References}

[1] O.C. Zienkiewicz, The Finite Element Method, third ed., Mc Graw-Hill, London, 1977.

[2] T.J.R. Hughes, The Finite Element Method, Static and Dynamic Analysis, Prentice Hall, Englewood Cliffs, N.J., 1987.

[3] K.J. Bathe, Finite Element Procedures, Prentice Hall, Englewood cliffs, N.J, 1996.

[4] P.C. Hammer, O.J. Marlowe, A.H. Stroud, Numerical integration over simplexes and cones, Math. Tables Other Aids Comput. 10 (1956) 130-136.

[5] P.C. Hammer, A.H. Stroud, Numerical integration over simplexes, Math. Tables Other Aids Comput. 10 (1956) $137-139$.

[6] P.C. Hammer, A.H. Stroud, Numerical evaluation of multiple integrals, Math. Tables Other Aids Comput 12 (1958) $272-280$.

[7] G.R. Cowper, Gaussian quadrature formulas for triangle, Int. J. Numer. Meth. Engng. 7 (1973) 405-408.

[8] J.N. Lyness, D. Jespersen, Moderate degree symmetric quadrature rules for the triangle, J. Inst. Math. Appl. 15 (1975) 19-32.

[9] F.G. Lannoy, Triangular finite elements and numerical integration, Comput. Struct. 7 (1977) 613.

[10] D.P. Laurie, Automatic numerical integration over a triangle, CSIR Spec. Rep. WISK 273, National Research Institute of Mathematical Sciences, Pretoria, 1977.

[11] M.E. Laursen, M. Gellert, Some criteria for numerically integrated matrices and quadrature formulas for triangles, Int. J. Numer. Meth. Engng. 12 (1978) 67-76.

[12] D.A. Dunavant, High degree efficient symmetrical Gaussian quadrature rules for the triangle, Int. J. Numer. Meth. Engng 21 (1985) 1129-1148.

[13] P. Sylvester, Symmetric Quadrature Formulae for Simplexes, Math. Comput. 24 (1970) 95-100.

[14] F.G. Lether, Computation of double integrals over a triangle, J. Comp. Appl. Math. 2 (1976) 219-224. 
[15] P. Hillion, Numerical integration on a triangle, Int. J. Numer. Meth. Engng. 11 (1977) 797-815.

[16] M. Abramowicz, I.A. Stegun, Hand Book of Mathematical Functions, Dover Publications, Inc., New York, 1965.

[17] C.T. Reddy, Improved three point integration schemes for triangular finite elements, Int. J. Numer. Meth. Engng. 12 (1978) 18901896.

[18] C.T. Reddy, D.J. Shippy, Alternative integration formulae for triangular finite elements, Int. J. Numer. Meth. Engng. 17 (1981) 133153.

[19] G. Lague, R. Baldur, Extended numerical integration method for triangular surfaces, Int. J. Numer. Meth. Engng. 11 (1977) 388-392.

[20] H.T. Rathod, H.S. Govinda Rao, Integration of polynomials over an arbitrary tetrahedron in Euclidean three dimensional space, Comput. Struct. 59 (1) (1996) 55-65.

[21] H.T. Rathod, H.S. Govinda Rao, Integration of trivariate polynomials over linear poly-hedra in Euclidean three dimensional space, J. Aust. Math. Soc. Ser. B 39 (1998) 355-385.

[22] H.T. Rathod, K.V. Nagaraja, B. Venkatesudu, N.L. Ramesh, Gauss Legendre quadrature over a triangle, J. Ind. Inst. Sci. 84 (2004) $183-188$

[23] H.T. Rathod, B. Venkatesudu, K.V. Nagaraja, Gauss Legendre quadrature formulas over a tetrahedron, Numer. Meth. Partial Diff. Eq. 22 (1) (2005) 197-219.

[24] C.F. Gauss, Methodus nova integratiam valores per approximationem inveniendi worke 3, 1814, pp. $163-196$.

[25] G. Szego, Orthogonal Polynomials, A.M.S Pub., 1959.

[26] J. Villadsen, M.L. Michelsen, Solution of differential equation models by polynomial approximation, Prentice Hall Inc, Englewood Cliffs, NJ, 1978. 